Gut and Liver, Vol. 11, No. 4, July 2017, pp. 449-450

EDITORIAL

\title{
Depressive Symptoms and Quality of Life in the Patients of Inflammatory Bowel Disease
}

\author{
Jung Won Lee \\ Department of Internal Medicine, Samsung Changwon Hospital, Changwon, Korea
}

\begin{abstract}
See "Association of Inflammatory Bowel Disease (IBD) with Depressive Symptoms in the United States Population and Independent Predictors of Depressive Symptoms in an IBD Population: A NHANES Study" by Sanjay Bhandari, et al. on page 512, Vol. 11. No. 4, 2017
\end{abstract}

Inflammatory bowel disease (IBD), Crohn's disease and ulcerative colitis, is a chronic relapsing inflammatory disorder of the intestine. IBD develops at the interaction between genetic predisposition leading to immunological abnormalities, environment and microbiome. ${ }^{1}$ It required considerable cost and lifelong medical need in most patients. ${ }^{2}$

IBDs can cause disabling conditions which negatively affect the psychological dimension of life including lower healthrelated quality of life as well as physical abnormality. ${ }^{3}$ There is a growing consensus that IBD is associated with depressionrelated symptoms. Psychological symptoms appear to be more prevalent during active disease states with no difference in prevalence between Crohn's disease and ulcerative colitis. ${ }^{4}$ Behavioral disturbances including depression-like symptoms have also been observed in patients with IBD. Many researches have demonstrated that psychological factors including depressive mood and anxiety might contribute more frequent IBD flare. ${ }^{5}$ And depression from the IBD patients might be associated with poor treatment compliance. Many systematic reviews revealed that patients with IBD have about a $20 \%$ prevalence rate of anxiety and a 15\% prevalence rate of depression. In detail, the prevalence of depressive disorder in IBD is 15\%; the prevalence of depressive symptoms is $22 \% .^{6}$ Although the exact prevalence of this comorbid relationship has not been systematically demonstrated to date, especially for population based study.

In this issue of Gut and Liver, the article "Association of inflammatory bowel disease (IBD) with depressive symptoms in the United States population and independent predictors of depressive symptoms in an IBD population: a NHANES study" performed population level epidemiologic study including 190,269,933 U.S. adults without IBD and 2,325,226 with IBD. ${ }^{7}$ The result showed that IBD and non-IBD population differed from each other. In detail, adults with IBD adults were likely to be overweight and have more comorbidities than those with non-IBD. Depressive symptom was present in 49\% of those with IBD versus 23\% in those without IBD (unadjusted odds ratio [OR], 3.1; 95\% confidence interval [CI], 1.6 to 6.1 ; $<<0.001$ ). On multivariate analysis, IBD was independently associated with depression (adjusted OR, 3.1; 95\% CI, 1.6 to 5.9; $p=0.002$ ). Those with 2 and $\geq 3$ comorbidities had higher risks of depression with OR of 1.6 and 2.4 as compared with those with no comorbidities, respectively. The factors which were protective of depression were older age (>51 years) and educational level of college degree or higher and higher income poverty ratio. Moreover, the factors associated with depression in IBD population were older age ( $>51$ years) and divorced/separated/widowed with partner. Among those who were depressed, 36\% of IBD versus $15 \%$ of non-IBD patients visited mental health professional or psychiatrist in the past 1 year $(\mathrm{p}=0.02)$. There was trend toward increased suicidal ideation in depressed IBD versus depressed non-IBD population ( $27 \%$ vs $12 \%, \mathrm{p}=0.08$ ).

The present study well utilized NHANES data with the goal of better understanding of prevalence. About $60 \%$ of U.S. population were included in the present study. Although this study based on self-reported history of IBD and eventually have recall bias, it might be sufficient to represent the actual cross sectional status of mental health problems of the patients with IBD in United States. Moreover, risk factors were concordant with

Correspondence to: Jung Won Lee

Department of Internal Medicine, Samsung Changwon Hospital, 158 Paryong-ro, Masanhoewon-gu, Changwon 51353, Korea

Tel: +82-55-290-1267, Fax: +82-55-233-5028, E-mail: saludos@naver.com

pISSN 1976-2283 eISSN 2005-1212 https://doi.org/10.5009/gnl17227

@) This is an Open Access article distributed under the terms of the Creative Commons Attribution Non-Commercial License (http://creativecommons.org/licenses/by-nc/4.0) which permits unrestricted non-commercial use, distribution, and reproduction in any medium, provided the original work is properly cited. 
previous meta-analysis study and the former results have more solid evidence base.

Depressive symptoms in the present study was significantly high compared than previous studies (49\% vs 22\%). It implies that significant mental health problems including depressive mood have been underestimated by their physicians and researchers. Composite scoring system such as Crohn's disease activity index (CDAI) and Mayo score do not sufficiently contain functional and emotional aspect of disease status. ${ }^{8}$ Over the past decade, health related quality of life measurement tools have been developed to estimate the range of functional limitations in patients with IBD. ${ }^{9}$ And U.S. Food and Drug Administration more preferred patients reported outcomes than classical composite scoring system. So, additional effort should be done toward establishing more comprehensive evaluation system which contained comprehensive patient emotional status regarding high prevalence of depressive symptoms of the present study.

Separated marital status and elderly patients with IBD are more likely to have depressive symptoms. Despite psychological interventions such as psychotherapy and patient education were not effective for the patients with IBD, it might be helpful for the patients with multiple risk factor with depressive symptoms. ${ }^{10}$ Based on the result of the present population based study, further studies are needed about the effect of psychotherapy, risk stratification and screening to improve emotional aspect as well as improving laboratory and endoscopy findings.

\section{CONFLICTS OF INTEREST}

No potential conflict of interest relevant to this article was reported.

\section{REFERENCES}

1. Lee JW, Im JP, Cheon JH, Kim YS, Kim JS, Han DS. Inflammatory bowel disease cohort studies in Korea: present and future. Intest Res 2015;13:213-218.

2. Ng WK, Wong SH, Ng SC. Changing epidemiological trends of inflammatory bowel disease in Asia. Intest Res 2016;14:111-119.

3. Graff LA, Walker JR, Bernstein CN. Depression and anxiety in inflammatory bowel disease: a review of comorbidity and management. Inflamm Bowel Dis 2009;15:1105-1118.

4. Bernstein CN. Psychological stress and depression: risk factors for IBD? Dig Dis 2016;34:58-63.

5. Levenstein S, Prantera C, Varvo V, et al. Stress and exacerbation in ulcerative colitis: a prospective study of patients enrolled in remission. Am J Gastroenterol 2000;95:1213-1220.

6. Neuendorf R, Harding A, Stello N, Hanes D, Wahbeh H. Depression and anxiety in patients with inflammatory bowel disease: a systematic review. J Psychosom Res 2016;87:70-80.

7. Bhandari S, Larson ME, Kumar N, Stein D. Association of inflammatory bowel disease (IBD) with depressive symptoms in the United States population and independent predictors of depressive symptoms in an IBD population: a NHANES study. Gut Liver 2017;11:512-519.

8. Peyrin-Biroulet L, Sandborn W, Sands BE, et al. Selecting Therapeutic Targets in Inflammatory Bowel Disease (STRIDE): determining therapeutic goals for treat-to-target. Am J Gastroenterol 2015;110:1324-1338.

9. Peyrin-Biroulet L, Panés J, Sandborn WJ, et al. Defining disease severity in inflammatory bowel diseases: current and future directions. Clin Gastroenterol Hepatol 2016;14:348-354.e17.

10. Timmer A, Preiss JC, Motschall E, Rücker G, Jantschek G, Moser G. Psychological interventions for treatment of inflammatory bowel disease. Cochrane Database Syst Rev 2011;(2):CD006913. 